\title{
VHDL generation from SDL specifications
}

Jean-Marc Daveau, Gilberto Fernandes Marchioro, Carlos Alberto Valderrama and Ahmed Amine Jerraya

TIMA laboratory

TIMA/INPG 46 Avenue Felix Viallet, 38031 Grenoble cedex, France

Email \{daveau, marchior, valderr, jerraya\}@verdon.imag.fr

$F A X:(+33) 476473814$

\begin{abstract}
The aim of this paper is to present an approach that allows the generation of VHDL from system level specifications in SDL. Our approach overcome the main known problem encountered by previous work which is the communication between different processes. We allow SDL communication to be translated into VHDL for synthesis. This is made possible by the use of an intermediate form that support a powerful communication model which enable the representation in a synthesis oriented manner of most communication schemes. This intermediate form allows the refinement of the system in order to obtain the desired solution. The main refinement step, called communication synthesis, is aimed at fixing the protocol and the interface used by the different processes to communicate. The refined specification is translated into VHDL for synthesis using existing CAD tools. We illustrate the feasibility of our approach through two SDL to VHDL translation examples.
\end{abstract}

\section{Keywords}

Hardware/Software codesign, SDL, System level specification, VHDL generation, Communication synthesis

\section{INTRODUCTION}

As the system complexity grows there is the need for new methods to handle large system design. One way to manage that complexity is to rise the level of abstraction of the specifications by using system level description languages. On the other side, as the level of abstraction rise the gap between the concepts used for the specifications at the system level (communication channels, interacting processes, data types) and those used for hardware synthesis becomes wider. Although these languages are well suited for the specification and validation of complex real time distributed systems, the concepts manipulated 
are not easy to map onto hardware description languages. It is thus necessary to defines methods for system level synthesis enabling efficient synthesis from system level specifications.

\subsection{Objective}

System level specification language offers concepts and methods adapted to the description of complex systems. They provide formal methods for verifying and validating the system behaviour. As a system level specification serves as a basis for deriving an implementation, it should abstract from implementation details in order to postpone implementation decisions and not to exclude any valid realisation. Therefore many intermediate refinement steps are needed to achieve a realisation. Each of this steps will fixes implementation details closing the gap between the specification and the realisation. Several concepts supported by system level specification languages (finite state machine, communication through high level schemes, exceptions) are not easily represented in hardware description languages and need a cumbersome implementation. Therefore, having an automatic translation is then an efficient and errors free way of deriving an implementation from system level specifications.

When translating high level languages for implementation the main problem is to convert high level communication model. Direct implementation of system communication models inevitably leads to infeasible solution as system communication models are abstract and general. To achieve efficient solutions different communication schemes and protocols may be needed in embedded systems as well as different interconnection topologies.

In this paper we introduce a new approach for generating VHDL code from SDL system level specifications that overcome the problem of mapping system level concepts into hardware. The main objective for our method are :

1. to have an automatic VHDL code generation from system level specifications. Concepts that have no efficient hardware semantics will not be handled. The code produced should be acceptable by existing simulation and synthesis tools.

2. to have an efficient implementation of system level communication schemes.

3. to be able to choose between different communication schemes through a library.

4. to be able to model the system behaviour independently of the communication in a modular way. System specification should be independent of the communication specification in order to allow changes in the communication scheme without any changes in the system specification.

In addition our methodology should be compatible with others system de- 
sign tools such as hardware/software partitioning and software code generation from SDL.

\subsection{Previous Work}

Several previous work on system level synthesis are reported in the literature [Gajski 95], [Narayan 91] but only a few of them address the field of system synthesis from SDL specification [Bonatti 95], [Glunz 93], [Pulkkinen 92]. Although SDL [ITU-T 93] is widely used in the software and telecommunication community [Bochmann 93], it did not gain acceptance among the hardware designers. Many approaches try to use or extend existing hardware description languages such as VHDL [Eles 94], OO-VHDL [Swamy 95] by adding some object oriented and high level communication features.

Several approaches have extended single threaded languages to support hardware and communication concepts. Most of them have used the $\mathrm{C}$ language such as HardwareC [Mooney 96] or $C^{X}$ [Ernst 93]. Another approach is to create a new system level specification language as SpecChart [Narayan 93], [Vahid 95].

Other approaches use synchronous specification languages [Halbwachs 93] such as Esterel [Boussinot 91] [Chiodo 96], Signal [Le Guernic 91], Alpha [Le Moenner 96] or StateCharts [Buchenrieder 96].

Only few approaches have tried to use existing distributed system specification language such as SDL [Bonatti 95], [Glunz 93], [Pulkkinen 92], Lotos [Carreras 96], [Delgado Kloos 95] or Estelle [Wytrebwicz 95]. This is mainly due to the gap existing between the concepts manipulated by such languages and those used in hardware description languages. These languages offers concepts like concurrency and abstract communication. We believe that this gap can be closed by the use of progressive refinements [Ben Ismail 95], [Krueger 92]. However most of the work has concentrated on straightforward translation. Specification refinements are needed as the abstraction level of system level specifications is often too high to be executed directly and efficiently. The goal in this phase is to produce an executable system that satisfies the high level specification and that also exhibits acceptable performances. This transformational phase can be thought as an interactive, human guided compilation. Human interaction is necessary because such automatic algorithms are beyond compiler technology [Krueger 92]. [Gajski 94], [Gong 96] uses SpecChart as an intermediate form for progressive refinements although this language does not allows to model easily complex communication schemes such as message passing.

[Bonatti 95], [Glunz 93] and [Pulkkinen 92] generate VHDL from SDL specification. In [Pulkkinen 92], strong restrictions to the communication model are made. The translation to VHDL does not provide a queue into which incoming signals of a process are stored (see section 2). Instead, it translates 
each SDL signal into a VHDL signal changing the communication model from message passing to signals (see section 3). Each signal can have at most one parameter. Although this approach allows an easy synthesis, only one implementation of the SDL communication model is possible. It provides a correct implementation of the SDL execution model only when each state of the finite state machine has one transition. In [Bonatti 95], the mapping algorithm also simplifies the communication model to obtain synthesisable hardware. Three protocols are available from the library :

1. one single position queue shared by all signals.

2. one single position queue for each signal. In this case a process may have several queues

3 . one $\mathrm{N}$ positions queue for all signals.

Any of the three protocols can be used and new protocols can be added to the library. As in [Pulkkinen 92] it assigns one VHDL signal for each SDL signal. When using the second protocol, the algorithm requires the designer interaction to assign priorities to signals. This model does not respect the SDL communication model as signals are consumed in the order of their priorities and not in the order of their arrival. Glunz [Glunz 93] presents a more powerful approach for SDL to VHDL translation. The communication model can be changed through the use of a protocol library but it support only a subset of SDL. It does not support transition in procedures, labels and doesn't seem to support states with different transitions fired by signals having a different number of parameters.

To our knowledge none of the previous approaches overcome the main encountered translation problems related to communication. The major contribution of this paper is to present an approach where the synthesis is made through an intermediate form which allows transformations and refinements on the system. This allows to support a wide subset of SDL and to overcome the problem of communication.

In the following sections we present our approach to system level synthesis from SDL specifications. The next section gives an overview of the main SDL concepts used for system specification. In section 3 we emphasize the main differences between SDL and VHDL concept that may cause translation problems. Section 4 presents our intermediate representation and the different design steps. Section 5 introduces the communication model used for intermediate refinement and the concept of communication units. In this section we also detail the steps needed to overcome the problem of communication in system level synthesis from high level communication specification. Section 6 describe synthesis from SDL and give the supported subset. Finally we present some results before concluding. 


\section{SYSTEM SPECIFICATIONS WITH SDL}

SDL (specification and description language) is intended for the modelling and simulation of real time, distributed and telecommunication systems and is standardised by the ITU [ITU-T 93]. A system described in SDL is regarded as a set of concurrent processes that communicate with each others using signals. SDL support different concepts for describing systems such as structure, behaviour and communication. SDL is intended for describing large designs at the system level. There are two SDL formats, a textual and a graphical one.

\subsection{Structure}

The static structure of a system is described by a hierarchy of blocks. A block can contain other blocks, resulting in a tree structure or a set of processes to describe the behaviour of a terminal block. Processes are connected with each other and to the boundary of the block by signalroutes. Blocks are connected together by channels. Channels and signalroutes are a way of conveying signals that are used by the processes to communicate. Signals exchanged by the processes follow a communication path made up of signalroutes and channels from the sending to the receiving process (Figure 2). SDL also support dynamic feature that are software oriented like dynamic process creation and dynamic addressing.

\subsection{Behaviour}

The behaviour of a system is described by a set of autonomous and concurrent processes. A process is described by a finite state machine that communicate asynchronously with other processes by signals. Each process has an input queue where signals are buffered on arrival. Signals are extracted from the input queue by the process in the order in which they arrived. In other words, signals are buffered in a first-in-first-out order.

Each process is composed of a set of states and transitions. The arrival of an expected signal in the input queue validate a transition and the process can then execute a set of actions such as manipulating variables, procedures call and emission of signals. The received signal determines the transition to be executed. When a signal has initiated a transition it is removed from the input queue. In SDL, variables are owned by a specific process and cannot be modified by others processes. The synchronisation between processes is achieved using the exchange of signals only. SDL includes communication through revealed and exported shared variables. However they are single-writer-multiplereaders. Shared variables are not recommended in the SDL92 standard. Each 
process has an unique address (Pid) which identify it. A signal always carries the address of the sending and the receiving processes in addition to possible values. The destination address may be used if the destination process cannot be determined statically and the address of the sending process may be used to reply to a signal. Figure 1 represents an SDL process specification. State start represents the default state. Input represents the guard of a transition. This transition will be triggered when the specified signal is extracted from the input queue. Task represent an action to perform when the transition is executed and output emit a signal with its possible parameters.

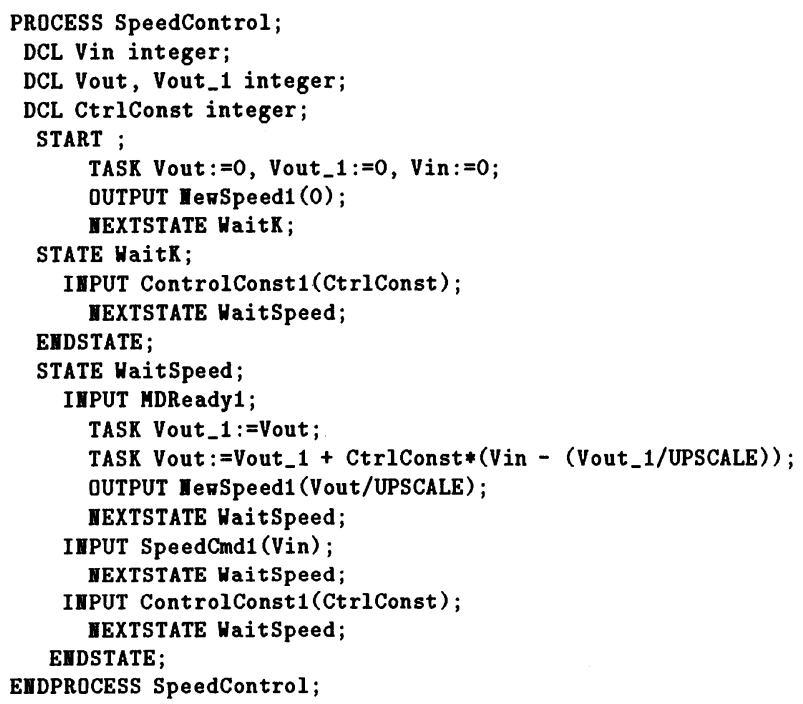

Figure 1 SDL process specification

\subsection{Communication}

Signals are transferred between processes using signalroutes and channels. If the processes are contained in different blocks, signals must traverse channels. The main difference between channels and signalroutes are :

- channels may perform a routing operation on signals, it routes a signal to different channels (signalroutes) connected to it at the frontier of a block depending on the receiving process.

- a communication through signalroutes is timeless while a communication through channel is delayed nondeterministically. No assumption can be made on the delay and no ordering can be presumed for signals using different delaying paths. 
Figure 2 represents the structure and communication specification of an SDL system.

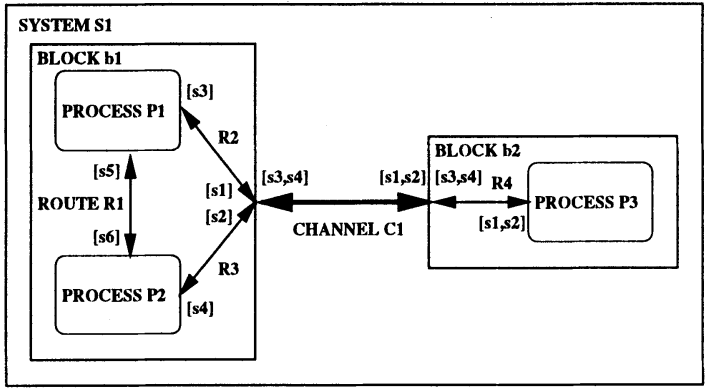

Figure 2 SDL signalroutes and channels

Channels and signalroutes may be both uni and bidirectional. If many signals are transferred on the same channel or signalroute their ordering is preserved. When going through a channel or signal route, signals are not allowed to overtake each other. However there is no specific ordering of signals arriving in the input queue of a process that have followed different channels and signalroutes.

\section{DISTANCE BETWEEN SDL AND VHDL CONCEPTS}

In this section we emphasize the main differences between SDL an VHDL concepts that may generate translation problems. These include :

\section{(a) Execution Model}

Every language is based on a computation model that specify the control or data flow and the synchronisation [Halbwachs 93]. SDL and VHDL are both control flow oriented.

In SDL, each process evolve independently according to the signals received. The execution of parallel processes can be non deterministic. For example when a process receive at the same time two signals coming from two different processes their ordering in the input queue cannot be predicted.

In VHDL, the execution of parallel processes is scheduled by the simulator. Every $\triangle$-cycle, all processes are evaluated. Signals assignement are instantaneous and deterministic as conflicts are resolved through resolution functions. With VHDL93 nondeterminism can be introduced using shared variables.

When going from an SDL specification to a VHDL implementation signals have to be scheduled in the input queues, one has then to ensure that the ordering of the signals is valid. 


\section{(b) Communication Abstraction}

Interprocess communication relies basically on two models : message passing and shared memory [Andrews 83]. SDL uses the former while VHDL uses the latter. In message passing, processes communicate through messages send with a specific protocol. With shared memory processes communicate through data stored in a common memory. In VHDL communication can also be carried using signals.

Modelling SDL communication in VHDL requires an expansion of the communication protocol and input queue that are implicit in SDL.

\section{(c) Behavioural Description}

The behavioural model specify how to describe the behaviour of the system.

In SDL, the basic construct is the finite state machine. SDL also provides dynamic features such as process creation or dynamic addressing.

In VHDL the number of component instances is fixed and behaviour is specified through processes in an algorithmic form. Moreover VHDL does not provide facilities for control flow specification such as exception, reset. Representing these concepts in VHDL requires cumbersome descriptions [Narayan 93].

\section{(d) Time}

In SDL, a time reference is offered by a global time server. This server manage the set of timers by providing set and reset primitives. Timers can be used to synchronise processes on an external event. The global time is available to every process through the variable now.

In VHDL, the time is also available through the global simulation time. Processes can use this global time for the synchronisation and the scheduling of signals assignement (for and after clause). The global simulation time is available through the variable now although it is not supported for synthesis. The translation of SDL timers implies choices concerning their realisation. In SDL the different timers are independent and are not synchronised by a common clock.

\section{(e) Data Type}

SDL offers the possibility to define abstract data types and generic operators for the specification of data types. Operators can be defined in an algorithmic form in SDL or C or through a state machine in SDL. VHDL only provides simple data types but SDL abstract data type can be translated in VHDL without introducing changes in the original program.

As shown in table 1, SDL support most of the system specification concepts as defined in [Vahid 95]. The main restrictions come from the behavioural hierarchy that is not supported and from a single available communication model. VHDL does not support directly some of these concepts (finite state machines, communication through high level schemes, exception handling) 


\begin{tabular}{|c|c|c|c|c|}
\hline language & hierarchy & concurrency & behaviour & timing \\
\hline SDL & structural & $\begin{array}{l}\text { single level } \\
\text { of process }\end{array}$ & $\begin{array}{l}\text { finite state } \\
\text { machines }\end{array}$ & timers \\
\hline VHDL & structural & $\begin{array}{c}\text { statement level } \\
\text { parallelism, } \\
\text { process }\end{array}$ & $\begin{array}{l}\text { algorithm, } \\
\text { data flow } \\
\text { equation }\end{array}$ & $\begin{array}{l}\text { AFTER } \\
\text { clause }\end{array}$ \\
\hline language & $\begin{array}{l}\text { exception } \\
\text { handling }\end{array}$ & synchronisation & communication & \\
\hline SDL & $\begin{array}{c}\text { yes } \\
\text { STATE * }\end{array}$ & global signals & $\begin{array}{l}\text { message passing, } \\
\text { FIFO protocol }\end{array}$ & \\
\hline VHDL & no & $\begin{array}{c}\text { WAIT } \\
\text { statement, } \\
\text { Common events }\end{array}$ & $\begin{array}{l}\text { shared memory, } \\
\text { signals }\end{array}$ & \\
\hline
\end{tabular}

Table 1 VHDL and SDL supported concepts

but they can be implemented using cumbersome description. A more complete comparison of several specification languages can be found in [Narayan 93].

\section{AN SDL BASED HARDWARE/SOFTWARE CODESIGN ENVIRONMENT}

In this section we present COSMOS, a methodology and an environment for the specification and the synthesis of mixed systems containing hardware and software. COSMOS starts with an SDL specification and produce a C/VHDL distributed architecture. In this paper we will concentrate on the VHDL generation only.

Our approach is based on an intermediate form on which interactive and incremental refinements are performed. The use of an intermediate representation permits the unification of different specifications described in hardware, software or system description languages. The codesign process starts with a functional specification which is translated into the intermediate form. The next steps are partitioning, communication synthesis and architecture generation.

The partitioning decompose the initial specification into abstract processors (partition) that may be transposed on the target architecture composed of hariware parts (ASIC, FPGA), software parts (processor + code) and communication modules (FIFO, memories, buses, IPC, interrupts). Each abstract processor may execute in hardware or in software.

Communication synthesis generally follows the system partitioning. Communication synthesis aims at fixing the protocol and interfaces used by the communicating subsystems. It is detailed in section 5. System are specified 
using the communication model offered by the specification language. This communication model may not suit the requirement of the final design and can be changed during communication synthesis.

The architecture generation step produces an executable description in $\mathrm{C}$ and VHDL for each abstract processor resulting of the partitioning step. The communication modules are extracted from a library.

\section{COMMUNICATION SYNTHESIS}

In this section we describe our communication modelling scheme for system level synthesis. This model aims at representing and implementing the communication scheme used in specification languages. Our model should be general enough to accommodate different communication schemes such as message passing or shared memory and allow an efficient implementation of a system level communication specifications.

\subsection{Communication Model}

In this paper we will use the communication modelling strategy described in [Ben Ismail 95]. At the system level, a system is represented by a set of

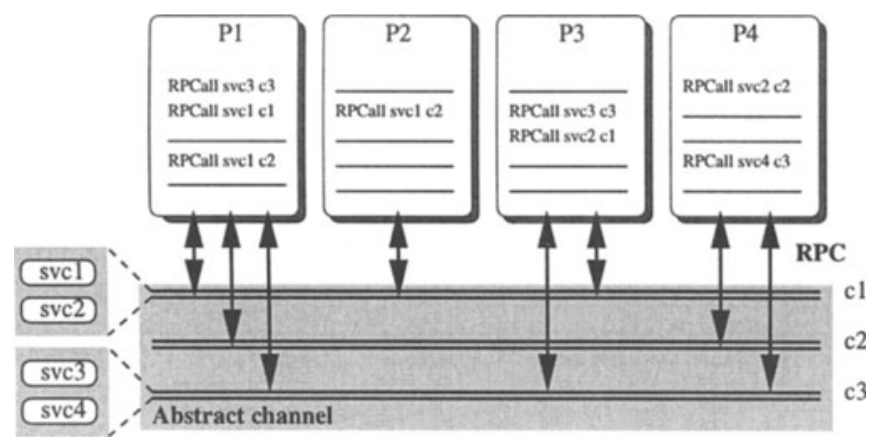

Figure 3 Specification of communication with abstract channels

processes communicating through abstract channels (figure 3). An abstract channel is an entity able to execute a communication scheme invoked through a procedure call mechanism. It offers high level communication primitives that are used by the processes to communicate. Access to the channel is controlled by this fixed set of communication primitives and relies on remote procedures call [Andrews 91] of these primitives. A process that is willing to communicate through a channel makes a remote procedure call to a communication primitive of that channel. Once the remote procedure call is done the com- 
munication is executed independently of the calling process by the channel unit. The communication primitives are transparent to the calling processes. This allows processes to communicate by means of high level communication schemes while making no assumption on the implementation of the communication.

There is no predefined set of communication primitives, they are defined as standard procedures and are attached to the abstract channel. Each application may have a different set of communication primitives (send_int, send_short, send_atm, etc). The communication primitives are the only visible part of an abstract channel.

The use of remote procedures call allows to separate communication specification from the rest of the system. These communication schemes can be described separately. In our approach the detailed I/O structure and protocols are hidden in a library of communication components. Figure 3 shows a conceptual communication over an abstract communication network. The processes communicate through three abstract channels $c 1, c 2$ and $c 3 . c 1$ and $c 2$ offers services $s v c 1, s v c 2$ and $c 3$ offers services $s v c 3, s v c 4$ (services $s v c 1$ and $s v c 2$ offered by abstract channel $c \mathscr{2}$ are not represented).

\subsection{Communication Unit Modelling}

We define a communication unit as an abstraction of a physical component. Communication units are selected from the library and instantiated during the communication synthesis step.

From a conceptual point of view, the communication unit is an object that can execute one or several communication primitives with a specific protocol. A communication unit is composed of a set of primitives, a controller and an interface. The complexity of the controller may range from a simple handshake to a complex layered protocol. This modular scheme hides the details of the realisation in a library where a communication unit may have different implementations depending on the target architecture (hardware/software).

Communication abstraction in this manner enables a modular specification, allowing communication to be treated independently from the rest of the design.

\subsection{Communication Synthesis}

Communication synthesis aims to transform a system composed of processes that communicate via high level primitives through abstract channels into a set of interconnected processors that communicate via signals and share communication control. Starting from such a specification two steps are needed. The first is aimed to fix the communication network structure and protocols 
used for data exchange. This step is called protocol selection or communication unit allocation. The second step, called interface synthesis, adapts the interface of the different processes to the selected communication network.

(a) Protocol Selection and Communication Unit Allocation Allocation of communication units starts with a set of processes communicating through abstract channels (figure 3 ) and a library of communication units (figure 4). These communication units are an abstraction of some physical
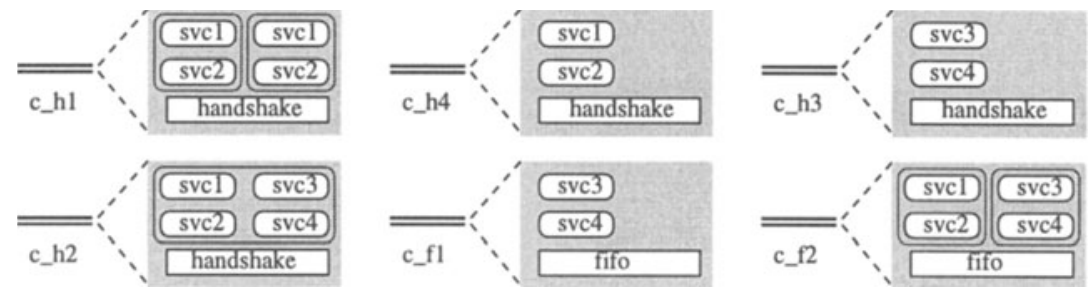

Figure 4 Library of communication units

components. This step chooses the appropriate set of communication units from the library in order to provide the services required by the communicating processes. The communication between the processes may be executed by one of the schemes described in the library. The choice of a given communication unit will not only depend on the communication to be executed but also on the performances required and the implementation technology of the communicating processes. This step fixes the protocol used by each communication primitive by choosing a communication unit with a specific protocol for each abstract channel. It also determines the interconnection topology of the processes by fixing the number of communication units and the abstract channels executed on it.

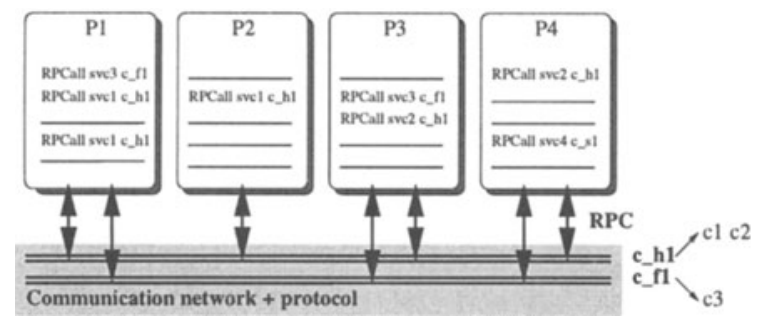

Figure 5 System after allocation of communication units

An example of communication unit allocation for the system of figure 3 is given in figure 5. Starting with the library of communication units of figure 4 , the communication unit $c_{-} h 1$ has been allocated for handling the communica- 
tion offered by the two abstract channels $c 1$ and $c 2$. Communication unit $c \_h 1$ is able to execute two independent communication requiring services svc1 and svc2. Communication unit $c_{-} f 1$ has been allocated for abstract channel $c 3$.

\section{(b) Interface Synthesis}

Interface synthesis selects an implementation for each of the communication units from the implementation library (figure 6) and generates the required interfaces and interconnections for all the processes using the communication units (figure 7). The library may contain several implementations of the same
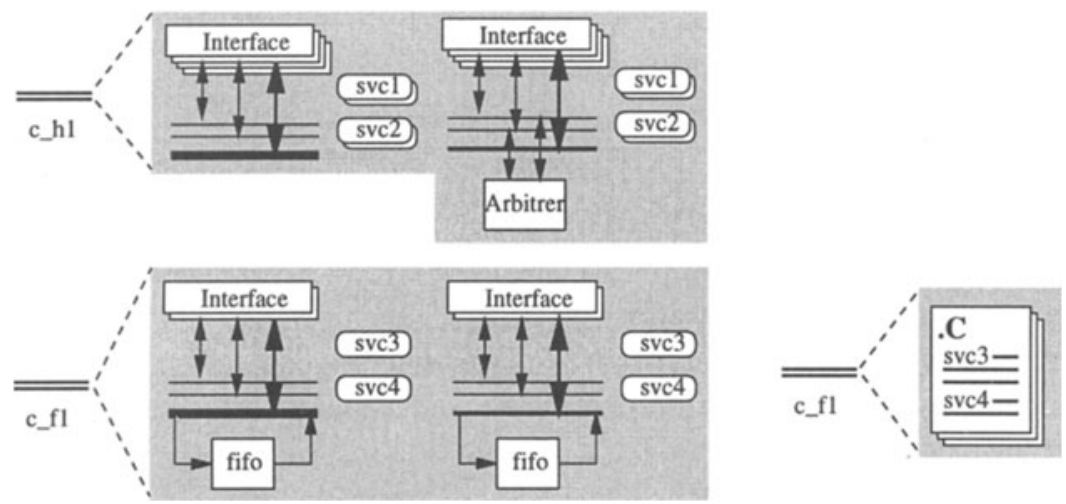

Figure 6 Implementation library

communication unit. Each communication unit is realised by a specific implementation selected from the library with regard to data transfer rates, memory buffering capacity, and the data bus width. The interface of the different processes are adapted according to the implementation selected and interconnected. The result of interface synthesis is a set of interconnected processors communicating through signals, buses and possible additional dedicated components selected from the implementation library such as bus arbiter, fifo, etc. With this approach it is possible to map communication specification into any protocol, from a simple handshake to a complex protocol.

Starting from the system of figure 5 , the result of interface synthesis task is detailed in figure 7 . The communication unit $c_{-} h 1$ has two possible implementations, one with an external bus arbiter for scheduling the two communication, the other with the arbiter distributed in the interfaces. Any of the two implementation may be selected.

\section{HARDWARE SYNTHESIS FROM SDL SPECIFICATIONS}

This section details the refinement steps and the models used by COSMOS in order to transform an SDL model into VHDL. This process support a large 


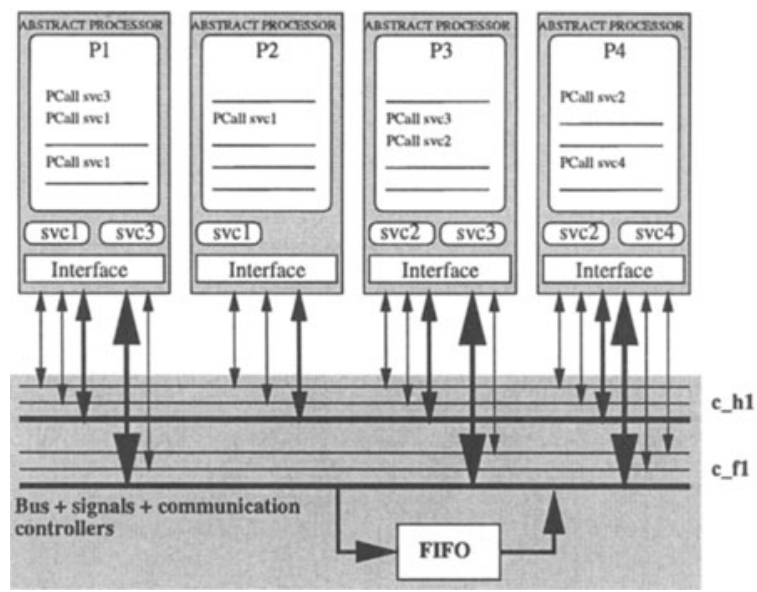

Figure 7 System after interface synthesis

subset of SDL. Only the concepts that are difficult to implement in hardware are excluded from the supported subset.

\subsection{Restriction for Hardware Synthesis}

SDL support a general and abstract communication model that is not well suited for hardware synthesis. This is mainly due to the fact that signals can be routed through channels. In other words the destination of a signal can be determined dynamically by the address of the receiver. In SDL, the dynamic routing scheme is mainly intended for use with the dynamic process creation feature. This feature is very software oriented and is difficult to map in hardware. Nevertheless we can restrict the SDL communication model for hardware synthesis without loosing too much of its generality and abstraction. The restriction imposed on SDL will concern its dynamical aspects such as process creation and message routing.

\subsection{Supported Subset}

A wide subset of SDL is supported including :

- system, block, process, multiple instances.

- state, state *, state *(), save, save *, continuous signals, enabling condition.

- input, output, signals with parameters, task, label, join, nextstate, stop, decision, procedure call, imported and exported variables. 
Feature not supported includes : dynamic creation of processes, Pid (supported for multiple instances processes), channel substructure, non determinism (input any, input none, decision any).

\subsection{Correspondence Model Between SDL and VHDL}

In this section we present the translation patterns for the main SDL concepts.

\section{(a) Structure}

As stated in paragraph 6.1 dynamic aspects of SDL are not considered for hardware synthesis. To avoid any routing problem and obtain an efficient communication, we will restrict ourselves to the case where the destination process of a signal can be statically determined. Communication structure can then be flattened at compile time. A signal emitted by a process through a set of channels must have a single receiver among the processes connected to these channels. In such a case, channels only forward signals from one boundary of a block to another. No routing decision may be taken as there is only one path for a signal through a set of channels. Therefore channels and signalroutes won't be represented in the final system. A process that is emitting a signal will write it directly in the input queue of the destination process without going through several channels. Flattening the communication eliminates the communication overhead that occurs when traversing several channels.

\section{(b) Behaviour}

Each SDL process will be translated into the corresponding finite state machine. During the partitioning step state machines may be splitted and merged to achieved the desired solution. This step may generate additional communication like shared variables. All the communication protocols and implementation details will be fixed by the communication synthesis step regardless from where it has been generated (initial specification or partitioning).

\section{(c) Communication}

In SDL, each process has a single implicit queue used to store incoming messages. Therefore we will associate one abstract channel to each process (figure 8). This abstract channel will stand for the input queue and will offer the required communication primitives. During the communication synthesis steps a communication unit able to execute the required communication scheme will be selected from the library. This approach allows the designer to choose from the library a communication unit that provide an efficient implementation of the required communication. Despite the fact that SDL offers only one communication model, several different protocols may be allocated from the library for different abstract channels (see paragraph 5.3.a).

Each signal will be translated as two communication primitives offered by 
the abstract channel to read and write the signal and its parameters in the channel.

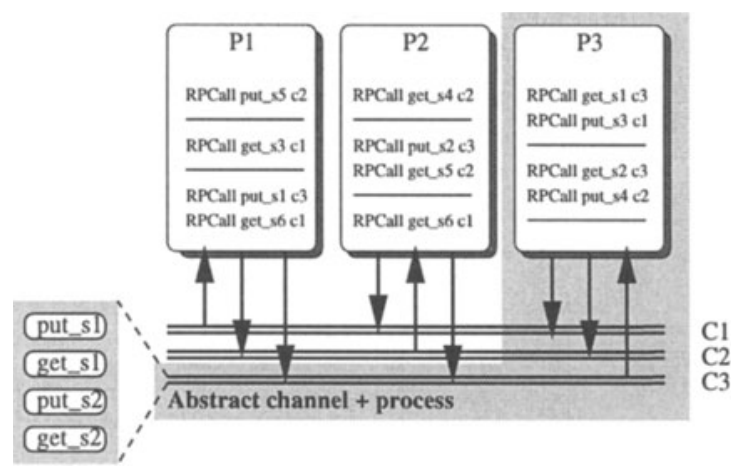

Figure 8 Modelling SDL communication with abstract channels

Figure 8 represents the refined SDL model corresponding to the system of figure 2 for synthesis. Each SDL process is mapped to a process containing the behavioural part of the specification and an abstract channel that offers communication primitives to send and receive signals. Each process will read from its own channel and write into other processes channel. An SDL specification is therefore represented by a set of processes and abstract channels. As stated before channels and signalroutes are not represented.

\section{RESULTS}

We present here some results concerning VHDL generation from system level specifications.

Figure 9 give the generated code for the process of figure 1. Figure 10 gives an implementation of the communication primitives implementing the SDL communication. The input queue of the process is not detailed in the example below. It is a standard fifo. Communication primitives (figure 10) read and write to the fifo ports.

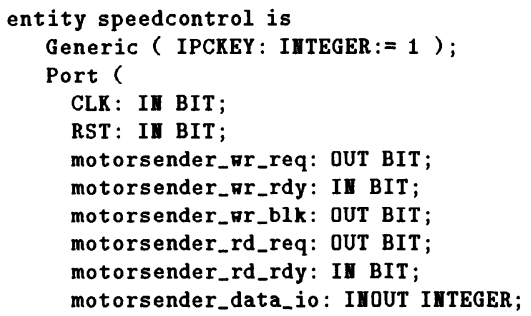



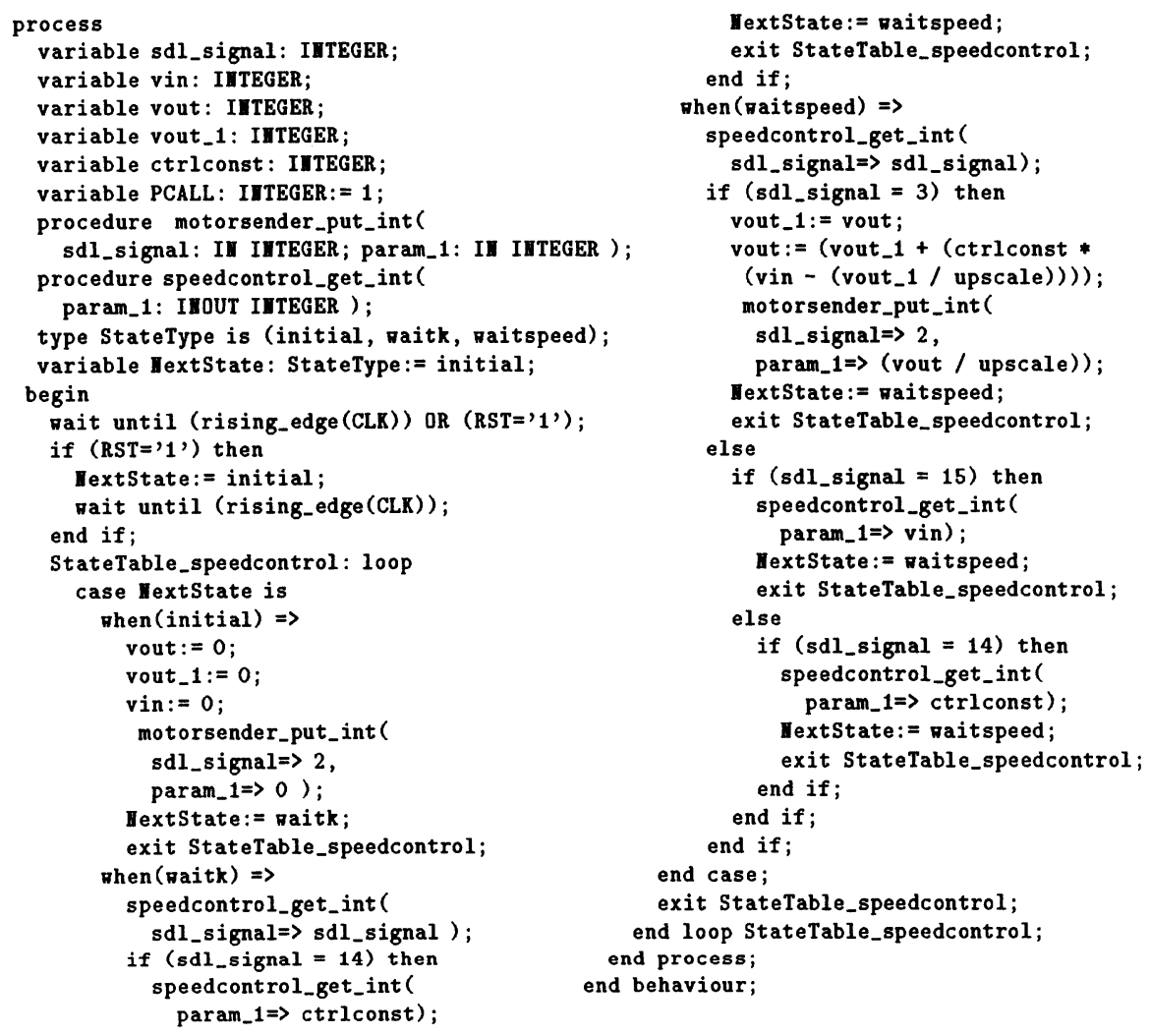

Figure 9 generated VHDL code

procedure motorsender_put_int(

sdl_signal: in IITEGER; param_1: in IITEGER) is

type StateType is (SPIDLE, request, signalio, param1io);

variable lext $S$ tate: $S$ tateType:= request;

begin

PCALL: $=1$;

phile (PCALL = 1) loop

StateTable_put_integer: loop

case MextState is

phen (request) $\Rightarrow$

motorsender_trr_req $<=$ ' 1 ; ;

motorsender_tr_blk $<=$, 1 ';

if $\operatorname{MoT}$ (motorsender_ar_rdy $=\prime_{1}^{\prime}$ ') then

rait until (motorsender_or_rdy $=\prime_{1}$ ');

end if;

motorsender_data_io< $=$ sdl_signal;

HextState:= signalio;

exit StateTable_put_integer;

phen (signalio) $\Rightarrow$

motorsender_ur_req $<=$ ' $O$ ';

if $\operatorname{HoT}$ (motorsender_wr_rdy $=, 0$ ') then

\section{קait until}

(motorsender_wr_rdy $={ }^{\prime} 0$ ) ); end if ;

motorsender_data_io $<=0$;

motorsender_ør_req $<=$ ',

if $\operatorname{nOT}$ (motorsender_kr_rdy

$=$ ' 1 ') then

rait until

(motorsender_wr_rdy $=$ '1 1 );

end if ;

motorsender_data_io $<=$ param_1;

motorsender_mr_blk $<=$ ' 0 ';

lextState: = param1io;

exit StateTable_put_integer;

when (param1io) $\Rightarrow$

motorsender_nr_req< $=$ ' 0 ';

if HOT (motorsender_mr_rdy

$=$ ' 0 ') then

wait until

(motorsender_ør_rdy $={ }^{\prime} 0 \prime$ '); end if ; 


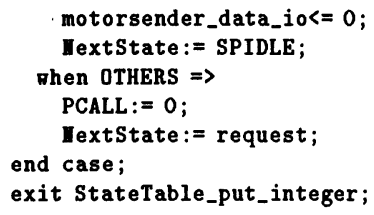

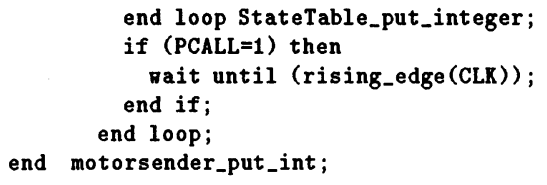

Figure 10 Implementation of communication primitives in VHDL

We can see from table 2 the line increase when going from SDL specification to VHDL implementation. Communication which represents only 10-20 $\%$ of the SDL specification represent more than $50 \%$ of the implementation in VHDL. This is mainly due to the the high level of abstraction of the communication provided by SDL. The VHDL size is more than seven times the size of the corresponding SDL model.

\begin{tabular}{ccccccc}
\hline \multirow{2}{*}{ design } & complexity & \multicolumn{2}{c}{ SDL lines } & \multicolumn{2}{c}{ VHDL lines } & lines \\
\cline { 3 - 6 } & $\begin{array}{c}\text { beha } \\
\text { viour }\end{array}$ & $\begin{array}{c}\text { commu } \\
\text { nication }\end{array}$ & $\begin{array}{c}\text { beha } \\
\text { viour }\end{array}$ & $\begin{array}{c}\text { commu } \\
\text { nication }\end{array}$ & increase \\
\hline $\begin{array}{c}\text { pid } \\
\text { controller }\end{array}$ & $\begin{array}{c}4 \text { processes } \\
34 \text { states } \\
33 \text { transitions }\end{array}$ & 331 & $\begin{array}{c}73 \\
(22 \%)\end{array}$ & 2403 & $\begin{array}{c}1194 \\
(50 \%)\end{array}$ & $726 \%$ \\
\hline $\begin{array}{c}\text { fuzzy logic } \\
\text { controller }\end{array}$ & $\begin{array}{c}9 \text { processes } \\
16 \text { states } \\
29 \text { transitions }\end{array}$ & 560 & $\begin{array}{c}88 \\
(15 \%)\end{array}$ & 4765 & 2856 & $850 \%$ \\
\hline
\end{tabular}

Table 2 SDL to VHDL translation results

In SDL, communication requires one instruction to send a signal. The protocol, signal conveying and input queue are implicit. When going at the implementation level communication becomes explicit requiring a specific protocol, communication controller and buses.

\section{CONCLUSION}

In this paper we have described an approach whereby the Specification and Description Language (SDL) can be used for system level synthesis to produce an implementation in VHDL. We have emphasised on the necessity of interactive and progressive refinement steps to close the gap between the concepts used in system description languages and those of hardware description language. Our approach support a large subset of SDL, i.e all concepts that may be used for hardware specification. The generated VHDL code is acceptable by existing simulation and high level synthesis tools. Efficient implementation of system communication is achieved through a library of communication models. 


\section{ACKNOWLEDGEMENTS}

This work was supported by France-Telecom/CNET under grant 94 1B 113, ESPRIT programme under project COMITY 23015, SGS-Thomson and Aerospatiale. The SDL to Solar translator was developed using the Verilog GEODE SDL environnement.

\section{REFERENCES}

G.R. Andrews and F.B. Schneider, Concepts and Notation for Concurrent Programming, Computing Survey, Vol 15, No 1, March 1983.

G.R. Andrews, Concurrent Programming, Principles and Practice, Benjamin/Cummings (eds), Redwood City, Calif., 1991.

T. Ben Ismail and A.A. Jerraya, Synthesis Steps and Design Models for CoDesign, IEEE Computer, special issue on rapid-prototyping of microelectronic systems, Vol. 28, No. 2, February 1995.

G.V. Bochmann, Specification Languages for Communication Protocols, Proceedings of the Conference on Hardware Description Languages, April 1993.

I.S. Bonatti and R.J.O. Figuerido, An Algorithm for the translation of SDL into Synthesizable VHDL, Current Issue in Electronic Modelling, Vol 3, August 1995.

F. Boussinot and R. De Simone, The ESTEREL Language, Proceedings of the IEEE, Vol. 79, No. 9, September 1991.

K. Buchenrieder, A. Pyttel and C. Veith, Mapping StateCharts Models onto an FPGA Based ASIP Architecture, Proceedings of the European Design Automation Conference with Euro-VHDL, September 1996.

C. Carreras, J.C. López et all, A Codesign Methodology Based on Formal Specifications and High Level Estimation, Proceedings of the CODES/CASHE workshop, March 1996.

M. Chiodo, D. Engels et all, A case Study in Computer Aided Codesign of Embedded Controllers, Design Automation for Embedded Systems, Vol. 1, No. 1-2, January 1996.

C. Delgado Kloos, A. Marín López et all, From Lotos to VHDL, Current Issue in Electronic modelling, Vol. 3, September 1995.

P. Eles, Z. Peng and A. Doboli, VHDL System Level Specification and Partitioning in a Hardware/Software Co-Synthesis environment, Proceedings of International Workshop on Hardware/Software Codesign, April 1994.

R. Ernst, J. Henkel and T. Benner, Hardware/Software Co-Synthesis for Microcontrollers, IEEE Design \& Test of Computers, Vol. 10 No. 4, pp. 64-75, December 1993.

D. Gajski, F. Vahid and S. Narayan, A Design Methodology for Systems Specification Refinement, Proceedings of the European Design Automation 
Conference (EDAC), February 1994.

D. Gajski and F. Vahid, Specification and Design of Embedded Hardware/Software Systems, IEEE Design \& Test of Computers, Spring 1995.

W. Glunz, T. Kruse, T. Rossel and D. Monjau, Integrating SDL and VHDL for System Level Specification, Proceedings of the Conference on Hardware Description Languages, April 1993.

J. Gong and D. Gajski, Model Refinement For Hardware Software Codesign, Proceedings of the European Design \& Test Conference, March 1996.

P. Le Guernic, T. Gautier et all, Programming Real-Time Applications with SIGNAL, Proceedings of the IEEE, Vol. 79, No. 9, September 1991.

N. Halbwachs, Synchronous programming of reactive systems, Kluwer Academic Publishers, ISBN 0-7923-9311-2, 1993.

C. Krueger, Software reuse, ACM computer survey, Vol. 24, No. 2, June 1992.

V.J. Mooney, C.N. Coelho, T. Sakamoto and G. De Micheli, Synthesis From Mixed Specifications, Proceedings of the European Design Automation Conference with Euro-VHDL, September 1996.

P. Le Moenner, L. Perraudeau et all, Generating Regular Arithmetic Circuit with ALPHAHARD, Proceedings of International Conference on Massively Parallel Computing Systems, May 1996.

S. Narayan and D. Gajski, Features Supporting System-Level Specification in $H D L s$, Proceedings of the European Design Automation Conference with Euro-VHDL, September 1993.

S. Narayan, F. Vahid and D. Gajski, Translating System Specifications to VHDL, Proceedings of the European Design Automation Conference (EDAC), February 1991.

O. Pulkkinen and K. Kronlöf, Integration of SDL and VHDL for High Level Digital Design, Proceedings of the European Design Automation Conference with Euro-VHDL, September 1992.

S. Swamy, A. Molin, and B. Covnot, OO-VHDL Object Oriented Extension to VHDL, IEEE Computer, Vol. 28, No. 10, October 1995.

F. Vahid, S. Narayan and D. Gajski, SpecCharts: A VHDL Front End for Embedded Systems, IEEE Transactions on Computer-Aided Design of Integrated Circuits and Systems, Vol. 14, No. 6, June 1995.

J. Wytrebwicz and S. Budkowski, Communication Protocols Implemented in Hardware: VHDL Generation from Estelle, Current Issue in Electronic Modelling, Vol 3, September 1995.

ITU-T, Z.100 Functional Specification and Description Language, Recommendation Z.100 - Z.104, March 1993. 